\title{
Existence of solution for integral boundary value problems of fractional differential equations
}

Xueying Yue ${ }^{1}$ and Kemei Zhang ${ }^{1 *}$ (I)

"Correspondence: zhkm90@126.com

'School of Mathematical Sciences,

Qufu Normal University, Qufu,

People's Republic of China

\begin{abstract}
In this paper, we discuss the existence of positive solutions of fractional differential equations on the infinite interval $(0,+\infty)$. The positive solution of fractional differential equations is gained by using the properties of the Green's function, Leray-Schauder's fixed point theorems, and Guo-Krasnosel'skii's fixed point theorem. As an application, two examples are given to prove our conclusions.
\end{abstract}

Keywords: Positive solutions; Fractional differential equations; Leray-Schauder fixed point theorem; Guo-Krasnosel'skii's fixed point theorem

\section{Introduction}

In this article, we investigate the positive solutions for fractional differential equations with infinite-point boundary value conditions:

$$
\left\{\begin{array}{l}
D_{0^{+}}^{\alpha} u(t)+q(t) f(t, u(t))=0, \quad t \in(0,+\infty) \\
u^{i}(0)=0, \quad i=0,1,2, \ldots, n-3 \\
D_{0^{+}}^{\alpha-1} u(+\infty)=0 \\
D_{0^{+}}^{\alpha-2} u(0)=\sum_{i=1}^{\infty} \beta_{i} u\left(\xi_{i}\right)
\end{array}\right.
$$

where $1<n-1<\alpha \leq n, n=[\alpha]+1, D_{0^{+}}^{\alpha}$ is the Riemann-Liouville fractional derivative, $0<\xi_{1}<\xi_{2}<\cdots<\xi_{i}<\cdots<+\infty, \beta_{i} \geq 0, i=0,1,2, \ldots, n-3, f:[0,+\infty) \times[0,+\infty) \rightarrow$ $[0,+\infty)$.

Recently, many kinds of fractional differential equations have been generally studied, see [1-15]. As everyone knows, fractional differential equations are used in many fields, for instance, control theory, mechanics, polymer rheology and engineering, and so on, for the details, see [16-21]. From the references, we can obtain that many authors have used the cone expansion and cone compression fixed point theorem to prove their conclusions, see $[5,22-26]$. (http://creativecommons.org/licenses/by/4.0/), which permits unrestricted use, distribution, and reproduction in any medium, provided you give appropriate credit to the original author(s) and the source, provide a link to the Creative Commons license, and indicate if changes were made. 
In [4], the authors studied the following equations:

$$
\left\{\begin{array}{l}
D_{0^{+}}^{\alpha} u(t)+f\left(t, u(t), D_{0^{+}}^{\alpha} u(t)\right)=0, \quad t \in(0,+\infty) \\
u(0)=0 \\
D_{0^{+}}^{\alpha-1} u(\infty)=\int_{0}^{\tau} g_{1}(s) u(s) d s+a \\
D_{0^{+}}^{\alpha-2} u(0)=\int_{0}^{\tau} g_{2}(s) u(s) d s+b
\end{array}\right.
$$

where $2<\alpha \leq 3, R^{+}=[0,+\infty), f: R^{+} \times\left(R^{+}\right)^{2} \rightarrow R^{+}, f(t, u, v) \not \equiv 0$, disturbance parameters $a, b \in R^{+}, g_{1}, g_{2} \in L^{1}[0, \tau)$ are nonnegative.

$\mathrm{Li}$ et al. in [7] investigated the following fractional:

$$
\left\{\begin{array}{l}
D_{0^{+}}^{\alpha} u(t)+f(t, u(t))=0, \quad t \in(0,1), \\
u(0)=0 \\
D_{0^{+}}^{\beta} u(1)=\alpha D_{0^{+}}^{\beta} u(\xi),
\end{array}\right.
$$

where $1<\alpha \leq 2$ and $D_{0^{+}}^{\alpha}$ is the Riemann-Liouville fractional derivative. The existence results of positive solutions are gained by using fixed point theorems.

Liang and Zhang [23] considered the following fractional boundary value problem:

$$
\left\{\begin{array}{l}
D_{0^{+}}^{\alpha} u(t)+a(t) f(t, u(t))=0, \quad t \in(0,+\infty), \\
u(0)=u^{\prime}(0)=0 \\
D_{0^{+}}^{\alpha-1} u(+\infty)=\sum_{i=1}^{m-2} \beta_{i} u\left(\xi_{i}\right)
\end{array}\right.
$$

where $2<\alpha<3, D_{0^{+}}^{\alpha}$ is the Riemann-Liouville fractional derivative, $0<\xi_{1}<\xi_{2}<\cdots<$ $\xi_{m-2}<+\infty, \beta_{i} \geq 0, i=1,2, \ldots, m-2$, satisfies $0<\sum_{i=1}^{m-2} \beta_{i} \xi_{i}^{\alpha-1}<\Gamma(\alpha)$.

By using Banach contraction mapping principle, the writers gave some new results for existence and uniqueness of positive solutions for the above problem in [12]. As far as we can see, there are still very few papers (such as [22]) discussing the fractional coupled systems. To prove our conclusions, we put forward some necessary basic concepts in Sect. 2. In Sect. 3, we obtain the positive solutions of BVP (1.1) by using the relevant fixed point theorem. In Sect. 4, two examples are presented to verify our results.

\section{Preliminaries and correlative lemmas}

To prove our conclusion, we first introduce some basic concepts, see [5, 9].

Definition 2.1 ([9]) The Riemann-Liouville fractional integral of order $\alpha>0$ of a function $u: R^{+} \rightarrow R$ is given by

$$
I_{0^{+}}^{\alpha} u(t)=\frac{1}{\Gamma(\alpha)} \int_{0}^{t}(t-s)^{\alpha-1} u(s) d s
$$

Definition 2.2 ([9]) The Riemann-Liouville fractional derivative of order $\alpha>0$ of a function $u: R^{+} \rightarrow R$ is given by

$$
D_{0^{+}}^{\alpha} u(t)=D^{n} I_{0^{+}}^{n-\alpha} u(t)=\frac{1}{\Gamma(n-\alpha)}\left(\frac{d}{d t}\right)^{n} \int_{0}^{t}(t-s)^{n-\alpha-1} u(s) d s,
$$

where $n=[\alpha]+1$. 
Remark 2.1 ([1]) In this way, we need the following conclusions:

1. $D_{0^{+}}^{\alpha} t^{\beta-1}=\frac{\Gamma(\beta)}{\Gamma(\beta-\alpha)} t^{\beta-\alpha-1}, \alpha, \beta \geq 0$;

2. $D_{0^{+}}^{\alpha} t^{\alpha-j}=0, j=1,2, \ldots,[\alpha]+1$.

Lemma $2.1([9,12])$ For $\alpha>0$, the equality $D_{0^{+}}^{\alpha} u(t)=0$ is well-founded if and only if

$$
u(t)=\sum_{i=1}^{n} c_{i} t^{\alpha-i}
$$

where $c_{i} \in R, i=1,2, \ldots, n, n=[\alpha]+1$.

Lemma $2.2([9,12])$ If $u \in C\left(R^{+}\right)$and $D_{0^{+}}^{\alpha} u \in L^{1}\left(R^{+}\right)$, then

$$
I_{0^{+}}^{\alpha} D_{0^{+}}^{\alpha} u(t)=u(t)+c_{1} t^{\alpha-1}+c_{2} t^{\alpha-2}+\cdots+c_{n} t^{\alpha-n}
$$

where $c_{i} \in R, i=1,2, \ldots, n, n=[\alpha]+1$.

Lemma 2.3 Let $h \in L^{1}[0,+\infty)$, the following boundary value problem:

$$
\left\{\begin{array}{l}
D_{0^{+}}^{\alpha} u(t)+h(t)=0, \quad t \in(0,+\infty) \\
u^{i}(0)=0, \quad i=0,1,2, \ldots, n-3 \\
D_{0^{+}}^{\alpha-1} u(+\infty)=0 \\
D_{0^{+}}^{\alpha-2} u(0)=\sum_{i=1}^{\infty} \beta_{i} u\left(\xi_{i}\right)
\end{array}\right.
$$

where $1<n-1<\alpha \leq n, n=[\alpha]+1,0<\xi_{1}<\xi_{2}<\cdots<\xi_{i}<\cdots<+\infty, \beta_{i} \geq 0, i=0,1,2, \ldots, n-$ 3 , satisfies $0<\sum_{i=1}^{\infty} \beta_{i} \xi_{i}^{\alpha-2}<\Gamma(\alpha)$, has a unique solution

$$
u(t)=\int_{0}^{+\infty} G(t, s) h(s) d s
$$

where

$$
\begin{aligned}
& G(t, s)=G_{1}(t, s)+G_{2}(t, s), \\
& G_{1}(t, s)=\frac{1}{\Gamma(\alpha)} \begin{cases}t^{\alpha-1}-(t-s)^{\alpha-1}, & 0 \leq s \leq t<+\infty, \\
t^{\alpha-1}, & 0 \leq t \leq s<+\infty,\end{cases} \\
& G_{2}(t, s)=\frac{\sum_{i=1}^{\infty} \beta_{i} t^{\alpha-2}}{\Gamma(\alpha-1)-\sum_{i=1}^{\infty} \beta_{i} \xi_{i}^{\alpha-2}} G_{1}\left(\xi_{i}, s\right) .
\end{aligned}
$$

Proof From Lemma 2.2 and (2.1), we have

$$
u(t)=-\frac{1}{\Gamma(\alpha)} \int_{0}^{t}(t-s)^{\alpha-1} h(s) d s+c_{1} t^{\alpha-1}+c_{2} t^{\alpha-2}+\cdots+c_{n} t^{\alpha-n}
$$

From $u^{i}(0)=0, i=0,1,2, \ldots, n-3$, we know that $c_{3}=c_{4}=\cdots=c_{n}=0$,

$$
D_{0^{+}}^{\alpha-1} u(t)=-\int_{0}^{t} h(s) d s+c_{1} \Gamma(\alpha)
$$


and

$$
D_{0^{+}}^{\alpha-2} u(t)=-\int_{0}^{t}(t-s) h(s) d s+c_{1} \Gamma(\alpha) t+c_{2} \Gamma(\alpha-1)
$$

By the boundary conditions, we can get

$$
\left\{\begin{array}{l}
-\int_{0}^{+\infty} h(s) d s+c_{1} \Gamma(\alpha)=0 \\
c_{2} \Gamma(\alpha-1)=\sum_{i=1}^{\infty} \beta_{i}\left(-\frac{1}{\Gamma(\alpha)} \int_{0}^{\xi_{i}}\left(\xi_{i}-s\right)^{\alpha-1} h(s) d s+c_{1} \xi_{i}^{\alpha-1}+c_{2} \xi_{i}^{\alpha-2}\right)
\end{array}\right.
$$

then

$$
c_{1}=\frac{1}{\Gamma(\alpha)} \int_{0}^{+\infty} h(s) d s
$$

and

$$
c_{2}=\frac{\sum_{i=1}^{\infty} \beta_{i}\left[\frac{1}{\Gamma(\alpha)} \int_{0}^{+\infty} \xi_{i}^{\alpha-1} h(s) d s-\frac{1}{\Gamma(\alpha)} \int_{0}^{\xi_{i}}\left(\xi_{i}-s\right)^{\alpha-1} h(s) d s\right]}{\Gamma(\alpha-1)-\sum_{i=1}^{\infty} \beta_{i} \xi_{i}^{\alpha-2}} .
$$

So

$$
\begin{aligned}
u(t)= & -\frac{1}{\Gamma(\alpha)} \int_{0}^{t}(t-s)^{\alpha-1} h(s) d s+\frac{1}{\Gamma(\alpha)} \int_{0}^{+\infty} t^{\alpha-1} h(s) d s \\
& +\frac{\sum_{i=1}^{\infty} \beta_{i} t^{\alpha-2}\left[\frac{1}{\Gamma(\alpha)} \int_{0}^{+\infty} \xi_{i}^{\alpha-1} h(s) d s-\frac{1}{\Gamma(\alpha)} \int_{0}^{\xi_{i}}\left(\xi_{i}-s\right)^{\alpha-1} h(s) d s\right]}{\Gamma(\alpha-1)-\sum_{i=1}^{\infty} \beta_{i} \xi_{i}^{\alpha-2}} \\
= & \int_{0}^{+\infty} G_{1}(t, s) h(s) d s+\frac{\sum_{i=1}^{\infty} \beta_{i} t^{\alpha-2}}{\Gamma(\alpha-1)-\sum_{i=1}^{\infty} \beta_{i} \xi_{i}^{\alpha-2}} \int_{0}^{+\infty} G_{1}\left(\xi_{i}, s\right) h(s) d s \\
= & \int_{0}^{+\infty} G_{1}(t, s) h(s) d s+\int_{0}^{+\infty} G_{2}(t, s) h(s) d s \\
= & \int_{0}^{+\infty} G(t, s) h(s) d s,
\end{aligned}
$$

where $G(t, s), G_{1}(t, s)$, and $G_{2}(t, s)$ are defined by (2.3)-(2.5).

Lemma 2.4 The function $G(t, s)$ defined by (2.3) satisfies

(1) $G_{1}(t, s) \geq 0$ is continuous for all $t, s \in R^{+} \times R^{+}$;

(2) $0 \leq \frac{G_{1}(t, s)}{1+t^{\alpha-1}} \leq \frac{1}{\Gamma(\alpha)}$ for all $t, s \in R^{+} \times R^{+}$;

(3) $0 \leq \frac{G(t, s)}{1+t^{\alpha-1}} \leq \frac{L}{\Gamma(\alpha)}$ for all $t, s \in R^{+} \times R^{+}, L=1+\frac{\sum_{i=1}^{\infty} \beta_{i} \xi_{i}^{\alpha-2}}{\Gamma(\alpha-1)-\sum_{i=1}^{\infty} \beta_{i} \xi_{i}^{\alpha-2}}$.

Proof (1) From (2.4), we know $G_{1}(t, s)$ is continuous on $[0,+\infty) \times[0,+\infty)$, obviously $G_{1}(t, s) \geq 0$ for $s \geq t$. For $0 \leq s \leq t<+\infty$, we have

$$
t^{\alpha-1}-(t-s)^{\alpha-1}=t^{\alpha-1}\left(1-\left(\frac{t-s}{t}\right)^{\alpha-1}\right) \geq 0
$$

By (2.4), we know $G_{1}(t, s) \geq 0, t, s \in[0,+\infty)$.

(2) From (2.4), it is easy to show that $0 \leq \frac{G_{1}(t, s)}{1+t^{\alpha-1}} \leq \frac{1}{\Gamma(\alpha)}$; 
(3) From (2), we have

$$
0 \leq \frac{G_{1}(t, s)}{1+t^{\alpha-1}} \leq \frac{1}{\Gamma(\alpha)}
$$

Furthermore, we have

$$
0 \leq \frac{G_{1}\left(\xi_{i}, s\right)}{1+t^{\alpha-1}} \leq \frac{1}{\Gamma(\alpha)}
$$

Therefore

$$
0 \leq \frac{G(t, s)}{1+t^{\alpha-1}}=\frac{G_{1}(t, s)}{1+t^{\alpha-1}}+\frac{G_{2}(t, s)}{1+t^{\alpha-1}} \leq \frac{1}{\Gamma(\alpha)}+\frac{1}{\Gamma(\alpha)} \frac{\sum_{i=1}^{\infty} \beta_{i} \xi_{i}^{\alpha-2}}{\Gamma(\alpha-1)-\sum_{i=1}^{\infty} \beta_{i} \xi_{i}^{\alpha-2}}=\frac{L}{\Gamma(\alpha)}
$$

and

$$
L=1+\frac{\sum_{i=1}^{\infty} \beta_{i} \xi_{i}^{\alpha-2}}{\Gamma(\alpha-1)-\sum_{i=1}^{\infty} \beta_{i} \xi_{i}^{\alpha-2}} .
$$

Lemma 2.5 ([23], Leray-Schauder fixed point theorem) Let B be a bounded, nonempty, convex, and closed subset of Banach space $E$, and let $F: B \rightarrow E$ be a completely continuous operator with $F(B) \subset B$. Then $F$ has a fixed point in $B$.

Lemma 2.6 ([9]) Let $k>1$ and denote $\lambda(k)=\min \left\{\frac{1}{4 k^{2}\left(1+k^{\alpha-1}\right)}, \frac{1}{k^{\alpha-2}\left(1+k^{\alpha-1}\right)}\right\}$ for a fixed number $k \in R^{+}$. Then functions $G_{1}, G_{2}$, and $G$ defined by (2.3)-(2.5) satisfy:

$$
\begin{aligned}
& \min _{t \in\left[\frac{1}{k}, k\right]} \frac{G_{1}(t, s)}{1+t^{\alpha-1}} \geq \frac{1}{4 k^{2}\left(1+k^{\alpha-1}\right)} \cdot \sup _{t \in[0,+\infty)} \frac{G_{1}(t, s)}{1+t^{\alpha-1}}, \\
& \min _{t \in\left[\frac{1}{k}, k\right]} \frac{G_{2}(t, s)}{1+t^{\alpha-1}} \geq \frac{1}{k^{\alpha-2}\left(1+k^{\alpha-1}\right)} \cdot \sup _{t \in[0,+\infty)} \frac{G_{2}(t, s)}{1+t^{\alpha-1}}, \\
& \min _{t \in\left[\frac{1}{k}, k\right]} \frac{G(t, s)}{1+t^{\alpha-1}} \geq \lambda(k) \cdot \sup _{t \in[0,+\infty)} \frac{G(t, s)}{1+t^{\alpha-1}},
\end{aligned}
$$

where $t, s \in[0,+\infty)$.

Proof According to Lemma 3.3 in [17], (2.6) is established.

From the definition of $G_{2}$, we have

$$
\begin{aligned}
\min _{t \in\left[\frac{1}{k}, k\right]} \frac{G_{2}(t, s)}{1+t^{\alpha-1}} & =\min _{t \in\left[\frac{1}{k}, k\right]} \frac{t^{\alpha-2}}{1+t^{\alpha-1}} \cdot \frac{\sum_{i=1}^{\infty} \beta_{i} G_{1}\left(\xi_{i}, s\right)}{\Gamma(\alpha-1)-\sum_{i=1}^{\infty} \beta_{i} \xi_{i}^{\alpha-2}} \\
& \geq \frac{\frac{1}{k^{\alpha-2}}}{1+k^{\alpha-1}} \cdot \frac{\sum_{i=1}^{\infty} \beta_{i} G_{1}\left(\xi_{i}, s\right)}{\Gamma(\alpha-1)-\sum_{i=1}^{\infty} \beta_{i} \xi_{i}^{\alpha-2}} \\
& =\frac{\frac{1}{k^{\alpha-2}}}{1+k^{\alpha-1}} \cdot \sup _{t \in[0,+\infty)} \frac{t^{\alpha-2}}{1+t^{\alpha-1}} \cdot \frac{\sum_{i=1}^{\infty} \beta_{i} G_{1}\left(\xi_{i}, s\right)}{\Gamma(\alpha-1)-\sum_{i=1}^{\infty} \beta_{i} \xi_{i}^{\alpha-2}} \\
& \geq \frac{1}{k^{\alpha-2}\left(1+k^{\alpha-1}\right)} \cdot \sup _{t \in[0,+\infty)} \frac{G_{2}(t, s)}{1+t^{\alpha-1}}
\end{aligned}
$$

Thus, (2.6) holds. According to the definition of $\lambda(k)$, obviously (2.8) holds. 
Lemma 2.7 ([25]) Let $V=\{u \in E:\|u\|<l, l>0\}, V_{0}=\left\{\frac{u(t)}{1+t^{\alpha-1}}: u \in V\right\}$. If $V_{0}$ is equicontinuous in any finite subinterval of $[0,+\infty)$ and equiconvergent at infinity, then $V$ is relatively compact on $E$.

Lemma 2.8 ([26], Guo-Krasnosel'skii's fixed point theorem) Let E be a Banach space, $P \subset E$ be a cone, $\Omega_{1}, \Omega_{2}$ be two bounded open sets of $E$ centered at the origin with $\overline{\Omega_{1}} \subset \Omega_{2}$. Suppose that $A: P \cap\left(\overline{\Omega_{2}} \backslash \Omega_{1}\right) \rightarrow P$ is a completely continuous operator such that either

(i) $\|A x\| \geq\|x\|, \forall x \in P \cap \partial \Omega_{1}$ and $\|A x\| \leq\|x\|, \forall x \in P \cap \partial \Omega_{2}$, or

(ii) $\|A x\| \leq\|x\|, \forall x \in P \cap \partial \Omega_{1}$ and $\|A x\| \geq\|x\|, \forall x \in P \cap \partial \Omega_{2}$

holds. Then $A$ has at least one fixed point in $P \cap\left(\overline{\Omega_{2}} \backslash \Omega_{1}\right)$.

In this article, we use the following space $E$ to study (1.1), which is denoted by

$$
E=\left\{u \in C[0,+\infty): \sup _{t \in R^{+}} \frac{|u(t)|}{1+t^{\alpha-1}}<+\infty\right\}
$$

with the norm $\|u\|=\sup _{t \in R^{+}} \frac{|u(t)|}{1+t^{\alpha-1}}$. Obviously, $(E,\|\cdot\|)$ is a Banach space.

Let $P=\left\{u \in E: u(t) \geq 0, t \in R^{+}\right\}, P \subset E$ is a cone of $E$.

Denote $P_{r}=\{u \in P: 0 \leq\|u\|<r\}, \partial P_{r}=\{x \in P:\|x\|=r\}$ and $\overline{P_{r}}=\{x \in P:\|x\| \leq r\}$ where $r>0$.

\section{Main results}

Define an operator $T: P \rightarrow E$ as follows:

$$
T u(t)=\int_{0}^{+\infty} G(t, s) q(s) f(s, u(s)) d s
$$

For any $u \in P \subset E, t, s \in[0,+\infty)$, we have

$$
G(t, s) q(s) f(s, u(s)) \leq G(t, s) q(s) \varphi_{l}(s) \leq \frac{L}{\Gamma(\alpha)} q(s) \varphi_{l}(s) .
$$

So, for any $t_{0} \in[0,+\infty)$, we have $\left|\operatorname{Tu}(t)-\operatorname{Tu}\left(t_{0}\right)\right| \rightarrow 0$. Thus, $\operatorname{Tu}(t)=\int_{0}^{+\infty} G(t, s) q(s) f(s$, $u(s)) d s$ is convergent, then $T: P \rightarrow E$ is well defined. Therefore, the solution of BVP (1.1) is equivalent to the fixed point of operator $T$.

If the following conditions are established, then $f:[0,+\infty) \times[0,+\infty) \rightarrow[0,+\infty)$ is known as an $L^{1}$-Carathéodory function:

(1) for each $u \in[0,+\infty), t \mapsto f(t, u)$ is measurable on $t \in[0,+\infty)$;

(2) for a.e. $t \in[0,+\infty), u \mapsto f(t, u)$ is continuous on $u \in[0,+\infty)$;

(3) for each $r>0$, there exists $\varphi_{r} \in L^{1}[0,+\infty)$ with $\varphi_{r}(t) \geq 0$ on $t \in[0,+\infty)$ such that

$$
\left|f\left(t,\left(1+t^{\alpha-1}\right) u\right)\right| \leq \varphi_{r}(t), \quad \text { for all }\|u\| \leq r \text {, and a.e. } t \in[0,+\infty) \text {. }
$$

Throughout this article, we hypothesize that the condition holds:

$\left(H_{1}\right) q \in L^{1}\left(R^{+}\right)$is nonnegative and $\int_{0}^{\infty} q(s) \varphi_{r}(s) d s<\infty$ for any $r>0$.

Denote

$$
f^{0}=\limsup _{u \rightarrow 0^{+}} \sup _{t \in R^{+}} \frac{f\left(t,\left(1+t^{\alpha-1}\right) u\right)}{u}, \quad \rho=\frac{\Gamma(\alpha)}{L \int_{0}^{+\infty} q(s) d s} .
$$


Theorem 3.1 Assume that $\left(H_{1}\right)$ holds, $q(t) f(t, 0) \not \equiv 0$ in any subinterval $(0,+\infty)$, if $f^{0}<\rho$, then $B V P(1.1)$ has at least one positive solution.

Proof Step 1: We show that $T: P \rightarrow P$.

Obviously, $T u(t)$ is continuous with respect to $t \in R^{+}$. For any $u \in P \subset E$, there exists $l>0$ such that $\|u\| \leq l, f$ is an $L^{1}$-Carathéodory function. Since $\sup _{t \in R^{+}} \frac{u(t)}{1+t^{\alpha-1}}<+\infty$, then

$$
\frac{T u(t)}{1+t^{\alpha-1}}<\frac{L}{\Gamma(\alpha)} \int_{0}^{+\infty} q(s) \varphi_{l}(s) d s<+\infty
$$

So $T u \in E$. Because $G, f, q$ are nonnegative, in that way $T u(t) \geq 0$, which signifies $T u \in P$ for any $u \in P$.

Step 2: we prove that $T$ is a continuous operator.

Let $u_{n}, u \in P, n=1,2, \ldots$, such that $\left\|u_{n}-u\right\| \rightarrow 0$ as $n \rightarrow \infty$, that is, $\frac{u_{n}(t)}{1+t^{\alpha-1}} \rightarrow \frac{u(t)}{1+t^{\alpha-1}}$. Then there exists $r>0$ such that $\left\|u_{n}\right\| \leq r,\|u\| \leq r$. Since $f$ is an $L^{1}$-Carathéodory function, then

$$
\left|f\left(s, u_{n}(s)\right)-f(s, u(s))\right| \rightarrow 0, \quad n \rightarrow \infty,
$$

and

$$
\begin{aligned}
\left|f\left(s, u_{n}(s)\right)-f(s, u(s))\right| & =\left|f\left(s,\left(1+s^{\alpha-1}\right) \frac{u_{n}(s)}{1+s^{\alpha-1}}\right)-f\left(s,\left(1+s^{\alpha-1}\right) \frac{u(s)}{1+s^{\alpha-1}}\right)\right| \\
& \leq 2 \varphi_{r}(s) .
\end{aligned}
$$

By using the Lebesgue dominated convergence theorem, we get

$$
\int_{0}^{+\infty} q(s)\left|f\left(s, u_{n}(s)\right)-f(s, u(s))\right| d s \rightarrow 0, \quad n \rightarrow \infty
$$

So, we have

$$
\begin{aligned}
\frac{\left|T u_{n}(t)-T u(t)\right|}{1+t^{\alpha-1}} & =\left|\int_{0}^{+\infty} \frac{G(t, s)}{1+t^{\alpha-1}} q(s)\left(f\left(s, u_{n}(s)\right)-f(s, u(s))\right) d s\right| \\
& \leq \int_{0}^{+\infty} \frac{G(t, s)}{1+t^{\alpha-1}} q(s)\left|f\left(s, u_{n}(s)\right)-f(s, u(s))\right| d s \\
& \leq \frac{L}{\Gamma(\alpha)} \int_{0}^{+\infty} q(s)\left|f\left(s, u_{n}(s)\right)-f(s, u(s))\right| d s \rightarrow 0, \quad n \rightarrow \infty .
\end{aligned}
$$

Hence, $\left\|T u_{n}-T u\right\| \rightarrow 0$ as $n \rightarrow \infty$, thus $T$ is a continuous operator.

Step 3: $T$ is relatively compact.

Let $B \subset P$ be a nonempty bounded closed subset, that is, there exists $k>0$ such that $\|u\| \leq k$ for all $u \in B$, so there exists $\varphi_{k} \in L^{1}\left(R^{+}\right)$such that

$$
|f(s, u(s))|=\left|f\left(s,\left(1+s^{\alpha-1}\right) \frac{u(s)}{1+s^{\alpha-1}}\right)\right| \leq \varphi_{k}(s) .
$$


(1) For any $u \in B$,

$$
\begin{aligned}
\left|\frac{T u(t)}{1+t^{\alpha-1}}\right| & =\left|\int_{0}^{+\infty} \frac{G(t, s)}{1+t^{\alpha-1}} q(s) f(s, u(s)) d s\right| \\
& \leq \frac{L}{\Gamma(\alpha)} \int_{0}^{+\infty} q(s) \varphi_{k}(s) d s \\
& <+\infty .
\end{aligned}
$$

So, $T(B)$ is uniformly bounded.

For notational convenience, denote

$$
V_{1}=\left\{z: z=\frac{x(t)}{1+t^{\alpha-1}}, x \in T(B)\right\} .
$$

(2) For any $A>0$, let $I=[0, A]$ be a compact interval and $t_{1}, t_{2} \in I, t_{2}>t_{1}$.

$$
\begin{aligned}
\left|\frac{T u\left(t_{1}\right)}{1+t_{1}^{\alpha-1}}-\frac{T u\left(t_{2}\right)}{1+t_{2}^{\alpha-1}}\right| \leq & \int_{0}^{+\infty}\left|\frac{G_{1}\left(t_{1}, s\right)}{1+t_{1}^{\alpha-1}}-\frac{G_{1}\left(t_{2}, s\right)}{1+t_{2}^{\alpha-1}}\right| q(s) f(s, u(s)) d s \\
& +\frac{\sum_{i=1}^{\infty} \beta_{i}}{\Gamma(\alpha-1)-\sum_{i=1}^{\infty} \beta_{i} \xi_{i}^{\alpha-2}}\left|\frac{t_{1}^{\alpha-2}}{1+t_{1}^{\alpha-1}}-\frac{t_{2}^{\alpha-2}}{1+t_{2}^{\alpha-1}}\right| \\
& \times \int_{0}^{+\infty} G_{1}\left(\xi_{i}, s\right) q(s) f(s, u(s)) d s \\
\leq & \int_{0}^{+\infty}\left|\frac{G_{1}\left(t_{1}, s\right)}{1+t_{1}^{\alpha-1}}-\frac{G_{1}\left(t_{2}, s\right)}{1+t_{1}^{\alpha-1}}\right| q(s) \varphi_{k}(s) d s \\
& +\int_{0}^{+\infty}\left|\frac{G_{1}\left(t_{2}, s\right)}{1+t_{1}^{\alpha-1}}-\frac{G_{1}\left(t_{2}, s\right)}{1+t_{2}^{\alpha-1}}\right| q(s) \varphi_{k}(s) d s \\
& +\frac{\sum_{i=1}^{\infty} \beta_{i}}{\Gamma(\alpha)\left(\Gamma(\alpha-1)-\sum_{i=1}^{\infty} \beta_{i} \xi_{i}^{\alpha-2}\right)}\left|\frac{t_{1}^{\alpha-2}}{1+t_{1}^{\alpha-1}}-\frac{t_{2}^{\alpha-2}}{1+t_{2}^{\alpha-1}}\right| \\
& \times \int_{0}^{+\infty} q(s) \varphi_{k}(s) d s .
\end{aligned}
$$

On the other hand, we have

$$
\begin{aligned}
\int_{0}^{+\infty} & \left|\frac{G_{1}\left(t_{1}, s\right)}{1+t_{1}^{\alpha-1}}-\frac{G_{1}\left(t_{2}, s\right)}{1+t_{1}^{\alpha-1}}\right| q(s) \varphi_{k}(s) d s \\
\leq & \int_{0}^{t_{1}}\left|\frac{G_{1}\left(t_{1}, s\right)}{1+t_{1}^{\alpha-1}}-\frac{G_{1}\left(t_{2}, s\right)}{1+t_{1}^{\alpha-1}}\right| q(s) \varphi_{k}(s) d s \\
& +\int_{t_{1}}^{t_{2}}\left|\frac{G_{1}\left(t_{1}, s\right)}{1+t_{1}^{\alpha-1}}-\frac{G_{1}\left(t_{2}, s\right)}{1+t_{1}^{\alpha-1}}\right| q(s) \varphi_{k}(s) d s \\
& +\int_{t_{2}}^{+\infty}\left|\frac{G_{1}\left(t_{1}, s\right)}{1+t_{1}^{\alpha-1}}-\frac{G_{1}\left(t_{2}, s\right)}{1+t_{1}^{\alpha-1}}\right| q(s) \varphi_{k}(s) d s \\
\leq & \varphi_{k} \int_{0}^{t_{1}} \frac{\left(t_{1}^{\alpha-1}-t_{2}^{\alpha-1}\right)+\left(\left(t_{2}-s\right)^{\alpha-1}-\left(t_{1}-s\right)^{\alpha-1}\right)}{1+t_{1}^{\alpha-1}} q(s) d s \\
& +\varphi_{k} \int_{t_{1}}^{t_{2}} \frac{\left(t_{1}^{\alpha-1}-t_{2}^{\alpha-1}\right)+\left(t_{2}-s\right)^{\alpha-1}}{1+t_{1}^{\alpha-1}} q(s) d s
\end{aligned}
$$




$$
\begin{aligned}
& +\varphi_{k} \int_{t_{2}}^{+\infty} \frac{t_{1}^{\alpha-1}-t_{2}^{\alpha-1}}{1+t_{1}^{\alpha-1}} q(s) d s \\
& \rightarrow 0 \text { uniformly as } t_{1} \rightarrow t_{2} .
\end{aligned}
$$

Similarly, we have

$$
\int_{0}^{+\infty}\left|\frac{G_{1}\left(t_{2}, s\right)}{1+t_{1}^{\alpha-1}}-\frac{G_{1}\left(t_{2}, s\right)}{1+t_{2}^{\alpha-1}}\right| q(s) \varphi_{k}(s) d s \rightarrow 0 \quad \text { uniformly as } t_{1} \rightarrow t_{2}
$$

So, we have

$$
\left|\frac{T u\left(t_{1}\right)}{1+t_{1}^{\alpha-1}}-\frac{T u\left(t_{2}\right)}{1+t_{2}^{\alpha-1}}\right| \rightarrow 0 \quad \text { uniformly as } t_{1} \rightarrow t_{2}
$$

Therefore, $V_{1}$ is locally equicontinuous on $R^{+}$.

(3) For any $u \in B$, we have

$$
\int_{0}^{+\infty} q(s) f(s, u(s)) d s \leq \varphi_{k} \int_{0}^{+\infty} q(s) d s<+\infty
$$

and

$$
\begin{aligned}
\lim _{t \rightarrow \infty} & \frac{1}{1+t^{\alpha-1}} \int_{0}^{+\infty} G_{1}(t, s) q(s) f(s, u(s)) d s \\
= & \lim _{t \rightarrow \infty} \frac{t^{\alpha-1}}{1+t^{\alpha-1}} \int_{t}^{+\infty} q(s) f(s, u(s)) d s \\
& +\lim _{t \rightarrow \infty} \frac{1}{1+t^{\alpha-1}} \int_{0}^{t}\left(t^{\alpha-1}-(t-s)^{\alpha-1}\right) q(s) f(s, u(s)) d s \\
\leq & \varphi_{k} \lim _{t \rightarrow \infty} \int_{t}^{+\infty} q(s) d s \\
& +\varphi_{k} \lim _{t \rightarrow \infty} \int_{0}^{t} \frac{\left(t^{\alpha-1}-(t-s)^{\alpha-1}\right)}{1+t^{\alpha-1}} q(s) d s \\
= & 0
\end{aligned}
$$

since $\lim _{t \rightarrow \infty} \frac{\left(t^{\alpha-1}-(t-s)^{\alpha-1}\right)}{1+t^{\alpha-1}}=0$ and $\lim _{t \rightarrow \infty} \int_{t}^{+\infty} q(s) d s=0$.

Thus

$$
\begin{aligned}
\lim _{t \rightarrow \infty}\left|\frac{\operatorname{Tu}(t)}{1+t^{\alpha-1}}\right| \\
=\lim _{t \rightarrow \infty} \frac{1}{1+t^{\alpha-1}} \int_{0}^{+\infty} G(t, s) q(s) f(s, u(s)) d s \\
=\lim _{t \rightarrow \infty} \frac{1}{1+t^{\alpha-1}} \int_{0}^{+\infty} G_{1}(t, s) q(s) f(s, u(s)) d s \\
\quad+\lim _{t \rightarrow \infty} \frac{\sum_{i=1}^{\infty} \beta_{i} t^{\alpha-2}}{\left(1+t^{\alpha-1}\right)\left(\Gamma(\alpha-1)-\sum_{i=1}^{\infty} \beta_{i} \xi_{i}^{\alpha-2}\right)} \int_{0}^{+\infty} \frac{\xi_{i}^{\alpha-1}}{\Gamma(\alpha)} q(s) f(s, u(s)) d s \\
\quad-\lim _{t \rightarrow \infty}^{\infty} \frac{\sum_{i=1}^{\infty} \beta_{i} t^{\alpha-2}}{\left(1+t^{\alpha-1}\right)\left(\Gamma(\alpha-1)-\sum_{i=1}^{\infty} \beta_{i} \xi_{i}^{\alpha-2}\right)} \int_{0}^{\xi_{i}} \frac{\left(\xi_{i}-s\right)^{\alpha-1}}{\Gamma(\alpha)} q(s) f(s, u(s)) d s
\end{aligned}
$$




$$
\begin{aligned}
& \leq \frac{\sum_{i=1}^{\infty} \beta_{i} \xi_{i}^{\alpha-1}}{\Gamma(\alpha)\left(\Gamma(\alpha-1)-\sum_{i=1}^{\infty} \beta_{i} \xi_{i}^{\alpha-2}\right)} \int_{0}^{+\infty} q(s) \varphi_{k}(s) d s \\
& \quad-\frac{\sum_{i=1}^{\infty} \beta_{i}}{\Gamma(\alpha)\left(\Gamma(\alpha-1)-\sum_{i=1}^{\infty} \beta_{i} \xi_{i}^{\alpha-2}\right)} \int_{0}^{\xi_{i}}\left(\xi_{i}-s\right)^{\alpha-1} q(s) \varphi_{k}(s) d s \\
& <+\infty
\end{aligned}
$$

that is, for any $\varepsilon>0$, there exists $N>0$ such that, for all $t_{1}, t_{2}>N$ and $T u \in P$, we have

$$
\left|\frac{T u\left(t_{2}\right)}{1+t_{2}^{\alpha-1}}-\frac{T u\left(t_{1}\right)}{1+t_{1}^{\alpha-1}}\right|<\varepsilon
$$

Hence, $V_{1}$ is equiconvergent at infinity. So $T$ is a completely continuous operator.

Step 4: $T(B) \subset B$.

Because $f^{0}<\rho$, there exists $r_{1}>0$ such that $\frac{f\left(t,\left(1+t^{\alpha-1}\right) u\right)}{u}<\rho u<\rho r_{1}, t \in R^{+}$, and $u \in\left(0, r_{1}\right]$.

Set $B=\left\{u \in P:\|u\| \leq r_{1}\right\}$, then, for any $u \in B$,

$$
f(s, u(s))=f\left(s,\left(1+s^{\alpha-1}\right) \frac{u(s)}{1+s^{\alpha-1}}\right) \leq \rho \frac{u(s)}{1+s^{\alpha-1}} \leq \rho r_{1},
$$

and

$$
\begin{aligned}
\frac{T u(t)}{1+t^{\alpha-1}} & =\int_{0}^{+\infty} \frac{G(t, s)}{1+t^{\alpha-1}} q(s) f(s, u(s)) d s \\
& \leq \frac{L \rho r_{1}}{\Gamma(\alpha)} \int_{0}^{+\infty} q(s) d s \\
& =r_{1} .
\end{aligned}
$$

So $T(B) \subset B$. According to Lemma 2.5, we get that BVP (1.1) has at least one positive solution.

Theorem 3.2 $f$ is an $L^{1}$-Carathéodory function. Let $F(t, u)=f\left(t,\left(1+t^{\alpha-1}\right) u\right), r_{2}>r_{1}>0$, $k>1,\left(H_{1}\right)$ hold. Suppose that $F$ satisfies one of the following conditions:

(A1) $F\left(t, \frac{u}{1+t^{\alpha-1}}\right) \geq \frac{r_{1}}{d}$ for all $(t, u) \in\left[\frac{1}{k}, k\right] \times\left[0, r_{1}\right]$ and $F\left(t, \frac{u}{1+t^{\alpha-1}}\right) \leq \frac{r_{2}}{D}$ for all $(t, u) \in[0,+\infty] \times\left[0, r_{2}\right]$

(A2) $F\left(t, \frac{u}{1+t^{\alpha-1}}\right) \leq \frac{r_{1}}{d}$ for all $(t, u) \in[0,+\infty] \times\left[0, r_{1}\right]$ and $F\left(t, \frac{u}{1+t^{\alpha-1}}\right) \geq \frac{r_{2}}{D}$ for all $(t, u) \in\left[\frac{1}{k}, k\right] \times\left[0, r_{2}\right]$.

Then BVP (1.1) has at least one positive solution $u^{*}$ with $r_{1} \leq\left\|u^{*}\right\| \leq r_{2}$.

Proof Firstly, we assume that (A1) holds.

According to Theorem 3.1, $T: \overline{P_{r_{2}}} \backslash P_{r_{1}} \rightarrow P$ is a completely continuous operator. By Lemma 2.8, the proof is as follows.

Step 1 . Let $u \in \partial P_{r_{1}}, t \in[0,+\infty)$, then $\frac{u(t)}{1+t^{\alpha-1}} \leq r_{1}$. We get the following conclusion by using (A1):

$$
\begin{aligned}
\min _{t \in\left[\frac{1}{k}, k\right]} \frac{|(T u)(t)|}{1+t^{\alpha-1}} & \geq \int_{0}^{+\infty} \min _{t \in\left[\frac{1}{k}, k\right]} \frac{G(t, s)}{1+t^{\alpha-1}} q(s) f(s, u(s)) d s \\
& \geq \lambda(k) \int_{0}^{+\infty} \sup _{t \in[0,+\infty)} \frac{G(t, s)}{1+t^{\alpha-1}} q(s) f(s, u(s)) d s
\end{aligned}
$$




$$
\begin{aligned}
& \geq \lambda(k) \int_{0}^{+\infty} \frac{G(t, s)}{1+t^{\alpha-1}} q(s) f(s, u(s)) d s \\
& \geq \frac{\lambda(k)}{k^{\alpha-2}\left(1+k^{\alpha-1}\right) \Gamma(\alpha)} \int_{\frac{1}{k}}^{k} q(s) F\left(s, \frac{u(s)}{1+s^{\alpha-1}}\right) d s \\
& \geq \frac{r_{1}}{d} \cdot \frac{\lambda(k)}{k^{\alpha-2}\left(1+k^{\alpha-1}\right) \Gamma(\alpha)} \int_{\frac{1}{k}}^{k} q(s) d s \\
& =r_{1},
\end{aligned}
$$

which means

$$
\|T u\| \geq\|u\|, \quad u \in \partial P_{r_{1}} .
$$

Step 2. Let $u \in \partial P_{r_{1}}, t \in[0,+\infty)$, then $\frac{u(t)}{1+t^{\alpha-1}} \leq r_{2}$. Using (A1) again, we get

$$
\begin{aligned}
\frac{|(T u)(t)|}{1+t^{\alpha-1}} & =\int_{0}^{+\infty} \frac{G(t, s)}{1+t^{\alpha-1}} q(s) f(s, u(s)) d s \\
& \leq \frac{L}{\Gamma(\alpha)} \int_{0}^{+\infty} q(s) F\left(s, \frac{u(s)}{1+s^{\alpha-1}}\right) d s \\
& \leq \frac{r_{2}}{D} \cdot \frac{L}{\Gamma(\alpha)} \int_{0}^{+\infty} q(s) d s \\
& =r_{2}
\end{aligned}
$$

which means

$$
\|T u\| \leq\|u\|, \quad u \in \partial P_{r_{2}} .
$$

Therefore, from Lemma 2.8(i), we get a positive solution $u^{*}$ with $r_{1} \leq\left\|u^{*}\right\| \leq r_{2}$ of BVP (1.1).

Similarly, condition (A2) has the same conclusion when it is established.

\section{Example}

Example 4.1 Consider the problem

$$
\left\{\begin{array}{l}
D_{0^{+}}^{\frac{9}{2}} u(t)+e^{-t-t^{\frac{7}{2}}} u^{2}=0, \quad t \in(0,+\infty) \\
u^{i}(0)=0, \quad i=0,1,2, \ldots, n-3 \\
D_{0^{+}}^{\frac{7}{2}} u(+\infty)=0 \\
D_{0^{+}}^{\frac{5}{2}} u(0)=\sum_{i=1}^{\infty} \frac{2}{i^{2}} u\left(1-\frac{1}{i+1}\right)
\end{array}\right.
$$

Let $\alpha=\frac{9}{2}, q(t)=e^{-t}, f(t, u)=e^{-t^{\frac{7}{2}}} u^{2}, \beta_{i}=\frac{2}{i^{2}}, x i_{i}=1-\frac{1}{i+1}$. It is easy to calculate that $\sum_{i=1}^{\infty} \beta_{i} \xi_{i}^{\alpha-2} \approx 1.643, \Gamma(\alpha)=\Gamma\left(\frac{9}{2}\right) \approx 11.6319, \Gamma(\alpha-1)=\Gamma\left(\frac{7}{2}\right) \approx 3.3234$, where $\varphi_{r}(t)=r^{2}(1+$ $\left.t^{\frac{7}{2}}\right)^{2} e^{-t^{\frac{7}{2}}} \in L^{1}\left(R^{+}\right)$, we have $f\left(t,\left(1+t^{\frac{2}{2}}\right)\right) \leq \varphi_{r}(t)$ for $u \leq r$ and $t \in R^{+}$. 
Then

$$
\begin{aligned}
& \int_{0}^{+\infty} q(s) \varphi_{r}(s) d s<+\infty, \\
& L=1+\frac{\sum_{i=1}^{\infty} \beta_{i} \xi_{i}^{\alpha-2}}{\Gamma(\alpha-1)-\sum_{i=1}^{\infty} \beta_{i} \xi_{i}^{\alpha-2}} \approx 1.9777, \\
& \rho=\frac{\Gamma(\alpha)}{L \int_{0}^{+\infty} q(s) d s} \approx 5.8815, \\
& f^{0}=\limsup _{u \rightarrow 0^{+}} \sup _{t \in R^{+}} \frac{f\left(t,\left(1+t^{\frac{7}{2}}\right) u\right)}{u}=\limsup _{u \rightarrow 0^{+}} \sup _{t \in R^{+}} \frac{e^{-t^{\frac{7}{2}}}\left(1+t^{\frac{7}{2}}\right)^{2} u^{2}}{u}=0<\rho .
\end{aligned}
$$

According to Theorem 3.1, we get that (4.1) has a positive solution.

Example 4.2 Consider the following problem:

$$
\left\{\begin{array}{l}
D_{0^{+}}^{\frac{9}{2}} u(t)+\left(t^{0.5}+e^{2}\right)^{-1} e^{-\pi t}\left(1.302 \times 10^{7}+u(t)+\sin u(t)\right)=0 \\
u^{i}(0)=0, \quad i=0,1,2, \ldots, n-3 \\
D_{0^{+}}^{\frac{7}{2}} u(+\infty)=0 \\
D_{0^{+}}^{\frac{5}{2}} u(0)=\sum_{i=1}^{\infty} \frac{2}{i^{2}} u\left(1-\frac{1}{i+1}\right)
\end{array}\right.
$$

where $t \in(0,+\infty), \alpha=\frac{9}{2}, \Gamma\left(\frac{9}{2}\right) \approx 11.6319, \Gamma\left(\frac{7}{2}\right) \approx 3.3234, \beta_{i}=\frac{2}{i^{2}}, \xi_{i}=1-\frac{1}{i+1}(i=$ $0,1,2, \ldots, n-3)$, and $\sum_{i=1}^{\infty} \beta_{i} \xi_{i}^{\alpha-2} \approx 1.643$.

Let $q(t)=t^{-0.5} e^{-\pi t}$ and $f(t, u)=\frac{1}{1+t^{1.5}}\left(1.302 \times 10^{7}+u+\sin u\right)$. Obviously, $\int_{0}^{+\infty} q(s) d s=1$. So condition $\left(H_{1}\right)$ holds. Set $k=2$. By calculation, we have $L \approx 1.9777, \lambda \approx 4.9783 \times 10^{-3}$, $d \approx 6.2653 \times 10^{-6}$, and $D \approx 0.2008$. Choose $r_{1}=\frac{1}{2}$ and $r_{2}=8 \times 10^{7}$. Then we get

$$
\begin{aligned}
f(t, u) & =\frac{1}{1+t^{1.5}}\left(1.302 \times 10^{7}+u+\sin u\right) \\
& >1.2305 \times 10^{7}>\frac{r_{1}}{d} \approx 7.9804 \times 10^{4}
\end{aligned}
$$

for all $(t, u) \in\left[\frac{1}{2}, 2\right] \times\left[0, \frac{1}{2}\right]$ and

$$
\begin{aligned}
f(t, u) & =\frac{1}{1+t^{1.5}}\left(1.302 \times 10^{7}+u+\sin u\right) \\
& <1.0209 \times 10^{8}<\frac{r_{2}}{D} \approx 3.9841 \times 10^{8}
\end{aligned}
$$

for all $(t, u) \in[0,+\infty] \times\left[0,8 \times 10^{7}\right]$.

So, according to Theorem 3.2, problem (4.2) has at least one solution.

\section{Acknowledgements}

The authors are grateful to the referees for carefully reading the paper and for their comments and suggestions.

\section{Funding}

The paper is supported by the National Science Foundation of Shandong (No. ZR2016FM10) and by the National Natural Science Foundation of China (No. 11571197). 
Competing interests

The authors declare that they have no competing interests.

\section{Authors' contributions}

All authors contributed equally and significantly in writing this article. All authors read and approved the final manuscript.

\section{Publisher's Note}

Springer Nature remains neutral with regard to jurisdictional claims in published maps and institutional affiliations.

Received: 27 June 2018 Accepted: 24 September 2018 Published online: 27 September 2018

\section{References}

1. Kilbas, A.A., Srivastava, H.M., Trujillo, J.J.: Theory and Applications of Fractional Differential Equations. North-Holland Mathematic Studies. Elsevier, Amsterdam (2006)

2. Miller, K.S., Ross, B.: An Introduction to the Fractional Calculus and Fractional Differential Equations. Wiley, New York (1993)

3. Zhou, Y.: Theory of Fractional Differential Equations. World Scientific, Singapore (2014)

4. Li, X.C., Liu, X.P., Jia, M., Li, X., Zhang, S.: Existence of positive solutions for integral boundary value problems of fractional differential equations on infinite interval. Math. Methods Appl. Sci. 40, 1892-1904 (2010)

5. Zhang, K.M.: Nontrivial solutions of fourth-order singular boundary value problems with sign-changing nonlinear terms. Topol. Methods Nonlinear Anal. 40, 53-70 (2012)

6. Wang, G.: Explicit iteration and unbounded solutions for fractional integral boundary value problem on an infinite interval. Appl. Math. Lett. 47, 1-7 (2015)

7. Li, C.F., Luo, X.N., Zhou, Y.: Existence of positive solutions of the boundary value problem for nonlinear fractional differential equations. Comput. Math. Appl. 59, 1363-1375 (2010)

8. Guan, Y.L., Zhao, Z.Q., Lin, X.L.: On the existence of positive solutions and negative solutions of singular fractional differential equations via global bifurcation techniques. Bound. Value Probl. 2016, 141 (2016)

9. Li, X., Liu, X., Jia, M., Zhang, L.: The positive solutions of infinite-point boundary value problem of fractional differential equations on the infinite interval. Adv. Differ. Equ. 2017, 126 (2017)

10. Zhang, X.: Positive solutions for a class of singular fractional differential equation with infinite-point boundary value conditions. Appl. Math. Lett. 39, 22-27 (2015)

11. Liu, Y.: Existence and unboundedness of positive solution for singular boundary value problem on half-line. Appl. Math. Comput. 144, 543-556 (2003)

12. Zhang, K.M.: On sign-changing solution for some fractional differential equations. Bound. Value Probl. $2017,59(2017)$

13. Denton, Z., Ramirez, J.D.: Existence of minimal and maximal solutions to RL fractional integro-differential initial value problems. Opusc. Math. 37, 705-724 (2017)

14. Kumar, S., Kumar, D., Singh, J.: Fractional modelling arising in unidirectional propagation of long waves in dispersive media. Adv. Nonlinear Anal. 5, 383-394 (2016)

15. Lyons, J.W., Neugebauer, J.T.: Positive solutions of a singular fractional boundary value problem with a fractional boundary condition. Opusc. Math. 37, 421-434 (2017)

16. Gao, H., Han, X.: Existence of positive solutions for fractional differential equation with nonlocal boundary condition. Int. J. Differ. Equ. 2011, 10 (2011)

17. Liang, S., Zhang, J.: Existence of three positive solutions of m-point boundary value problems for some nonlinear fractional differential equations on an infinite interval. Comput. Math. Appl. 61, 3343-3354 (2011)

18. Smart, D.R.: Fixed Point Theorems. Cambridge University Press, Cambridge (1974)

19. Podlubny, I.: Fractional Differential Equations. Academic Press, San Diego (1999)

20. Liu, L.S., Li, H.D., Liu, C., Wu, Y.H.: Existence and uniqueness of positive solutions for singular fractional differential systems with coupled integral boundary value problems. J. Nonlinear Sci. Appl. 10, 243-262 (2017)

21. Lin, X.L., Zhao, Z.Q.: Sign-changing solution for a third-order boundary value problem in ordered Banach space with lattice structure. Bound. Value Probl. 2014, 132 (2014)

22. Wang, J., Xiang, H., Liu, Z.: Positive solution to nonzero boundary values problem for a coupled system of nonlinear fractional differential equations. Int. J. Differ. Equ. 2010, Article ID 186928 (2010)

23. Liang, S.H., Zhang, J.H.: Existence of multiple positive solutions for $\mathrm{m}$-point fractional boundary value problems on an interval. Math. Comput. Model. 54, 1334-1346 (2011)

24. Gao, Y., Chen, P.: Existence of solutions for a class of nonlinear higher-order fractional differential equation with fractional nonlocal boundary condition. Adv. Differ. Equ. 2016, 314 (2016)

25. Liu, Y.: Existence and unboundedness of positive solutions for singular boundary value problems on half-line. Appl. Math. Comput. 144, 543-556 (2003)

26. Guo, D., Lakshmikantham, V.: Nonlinear Problems in Abstract Cones. Academic Press, New York (1988) 